\title{
Neurofilament light as a blood biomarker for neurodegeneration in Down syndrome

Andre Strydom ${ }^{1,2,3^{*}}$ D, Amanda Heslegrave ${ }^{4}$, Carla M. Startin ${ }^{1,2,3}$, Kin Y. Mok ${ }^{3,4,5}$, John Hardy ${ }^{3,4,6}$, Jurgen Groet ${ }^{3,7}$, Dean Nizetic ${ }^{3,7,8}$, Henrik Zetterberg ${ }^{4,9,10}$ and The LonDownS Consortium

\begin{abstract}
Background: Down syndrome (DS) may be considered a genetic form of Alzheimer's disease (AD) due to universal development of AD neuropathology, but diagnosis and treatment trials are hampered by a lack of reliable blood biomarkers. A potential biomarker is neurofilament light (NF-L), due to its association with axonal damage in neurodegenerative conditions.

Methods: We measured blood NF-L concentrations in 100 adults with DS using Simoa NF-light ${ }^{\circledR}$ assays, and we examined relationships with age as well as cross-sectional and longitudinal dementia diagnosis.

Results: NF-L concentrations increased with age (Spearman's rho $=0.789, p<0.001$ ), with a steep increase after age 40 , and they were predictive of dementia status ( $p=0.022$ adjusting for age, sex, and APOE4), but they showed no relationship with long-standing epilepsy or premorbid ability. Baseline NF-L concentrations were associated with longitudinal dementia status.
\end{abstract}

Conclusions: NF-L is a biomarker for neurodegeneration in DS with potential for use in future clinical trials to prevent or delay dementia.

Keywords: Down syndrome, Alzheimer's disease, Dementia, Neurofilament light, Biomarker

\section{Background}

Down syndrome (DS), caused by the trisomy, translocation, or partial trisomy of chromosome 21 , is the most common genetic cause of intellectual disability (ID), with an estimated population prevalence of 6 million worldwide. Dementia is a common feature of the aging process in DS that is due to the triplication of the amyloid precursor protein on chromosome 21, leading to brain pathology indicative of Alzheimer's disease (AD) [1], with a cumulative incidence for dementia in excess of $90 \%$ by the age of 65 [2] and a mean age at dementia diagnosis of 55 [3]. $\mathrm{DS}$ is therefore a genetic form of $\mathrm{AD}$ alongside autosomal dominant causes of AD [4].

Neurofilament light (NF-L) is one of the scaffolding cytoskeleton proteins of myelinated subcortical axons [5] and can now be reliably measured in blood using

\footnotetext{
* Correspondence: andre.strydom@kcl.ac.uk

'Department of Forensic and Neurodevelopmental Sciences, Institute of Psychiatry, Psychology and Neuroscience, King's College London, 16 De Crespigny Park, London SE5 8AF, UK

${ }^{2}$ Division of Psychiatry, University College London, London, UK

Full list of author information is available at the end of the article
}

ultrasensitive single-molecule array (Simoa) technology. Blood concentration of NF-L correlates well with corresponding cerebrospinal fluid (CSF) measures [6] and reflects axonal damage in neurological disorders, including frontotemporal dementia [7], multiple sclerosis [8], and familial and sporadic AD [9, 10]. NF-L correlates with other measures of disease stage and severity [10], but the utility of NF-L in populations with other genetic forms of $\mathrm{AD}$ is yet to be fully explored.

Aging in $\mathrm{DS}$ is invariably associated with $\mathrm{AD}$ pathology, and this condition therefore presents an opportunity to confirm the relationship between NF-L and progression of $\mathrm{AD}$ pathology. Furthermore, DS is a critically important group for clinical trials of treatments to prevent and delay $\mathrm{AD}$ pathology and dementia symptoms, and a reliable blood biomarker would help to address disease burden in this vulnerable population by enabling clinical trials of disease-modifying treatments. In the present study, we aimed to explore the relationship between plasma NF-L levels, age, and dementia status in individuals with DS, as well as its independence 
from sex effects, premorbid intellectual ability levels, and long-standing epilepsy.

\section{Methods}

Participants aged 16 years and older were recruited across England via care homes, support groups, and local NHS sites. Participants with an acute physical or mental health condition were excluded until they had recovered; other details of the cohort have been described previously [11]. DS status was confirmed using DNA from saliva or blood and genotyped using OmniExpressExome arrays (Illumina, San Diego, CA, USA); trisomy status was visually confirmed in GenomeStudio software (Illumina) (see Table 1). Apolipoprotein E (APOE) status was determined using TaqMan assays for rs7412 and rs429358 (Thermo Fisher Scientific, Waltham, MA, USA).

Assessment included a detailed interview with carers using the Cambridge Examination of Mental Disorders of Older People with Down's syndrome and others with intellectual disabilities [12] to determine decline in several domains, including memory. Premorbid ID level was defined according to the International Classification of Diseases, Tenth Revision, diagnostic system's descriptions of mild, moderate, and severe ID, based on carers' reports of the individuals' best-ever level of functioning [13]. At baseline, dementia was defined as a confirmed clinical diagnosis. At follow-up, participants were classified according to whether they had retained or been given a diagnosis of dementia or were being investigated for dementia.

Blood samples from 100 individuals were collected in lithium heparin tubes (Fisher Scientific UK, Loughborough, UK) and sent overnight for processing. Blood was layered over a similar amount of Ficoll (GE Healthcare, Little
Chalfont, UK), then centrifuged in a swing-out rotor for 40 minutes at $400 \times g$ without braking. Plasma samples were stored at $-80{ }^{\circ} \mathrm{C}$. Plasma NF-L concentration was measured by the same laboratory technician with reagents from a single lot using the Simoa NF-light ${ }^{\circ}$ assay (a digital sandwich immunoassay employing antibodies directed against the rod domain of NF-L) on an HD-1 Simoa analyzer according to the protocol issued by the manufacturer (Quanterix, Lexington, MA, USA). Samples were run in duplicate, and coefficients of variation (CVs) for duplicates were set to be $<12 \%$. All samples measured within the range spanned by the limits of quantification and interassay CV for the high- and low-concentration quality controls were $6.6 \%$ and $8.1 \%$, respectively.

All statistical tests were two-sided, and statistical significance was set at $p<0.05$. We tested associations between plasma NF-L samples and demographic or clinical factors using Mann-Whiney $U$, Kruskal-Wallis, and Spearman's rank correlation tests as appropriate. Associations between plasma NF-L and dementia diagnosis were tested using logistic regression and log-transformed NF-L values, with adjustment for age and sex; we also adjusted for APOE4 status cross-sectionally.

\section{Results}

NF-L levels were obtained from 100 participants (age range 17-73 years). Five results were excluded after failing to meet $\mathrm{CV}$ thresholds, meaning 95 adults were included in subsequent analyses. Of adults aged 36 and older who are being targeted for longitudinal follow-up, 29 of $63(46 \%)$ had completed a follow-up assessment at the time of this report (mean number of months between assessments 23.4, SD 3.9). One individual had

Table 1 Demographics of all participants included in group and subgroup analyses

\begin{tabular}{|c|c|c|c|c|}
\hline & All participants & Dementia (baseline) & No dementia (baseline) & Participants with follow-up data \\
\hline Number of subjects & 94 & 18 & 76 & 29 \\
\hline $\begin{array}{l}\text { Age at baseline, years, } \\
\text { mean } \pm \text { SD (range) }\end{array}$ & $42.68 \pm 14.87(17-73)$ & $55.17 \pm 9.92(40-69)$ & $39.72 \pm 14.34(17-73)$ & $52.63 \pm 8.88(40-72)$ \\
\hline DS type & $\begin{array}{l}89(94.7 \%) \text { trisomy, } 2(2.1 \%) \\
\text { translocation, } 3(3.2 \%) \\
\text { unknown }\end{array}$ & 18 (100.0\%) trisomy & $\begin{array}{l}71(93.4 \%) \text { trisomy, } 2 \\
(2.6 \%) \text { translocation, } 3 \\
(3.9 \%) \text { unknown }\end{array}$ & $\begin{array}{l}28(96.6 \%) \text { trisomy, } 1 \text { (3.4\%) } \\
\text { unknown }\end{array}$ \\
\hline Sex & $\begin{array}{l}41(43.6 \%) \text { female, } 53 \\
(56.4 \%) \text { male }\end{array}$ & $\begin{array}{l}6(33.3 \%) \text { female, } 12(66.7 \%) \\
\text { male }\end{array}$ & $\begin{array}{l}35(46.1 \%) \text { female, } 41 \\
(55.9 \%) \text { male }\end{array}$ & $\begin{array}{l}10(34.5 \%) \text { female, } 19(65.5 \%) \\
\text { male }\end{array}$ \\
\hline Ethnicity & $\begin{array}{l}85(90.4 \%) \text { white, } 9 \text { (9.6\%) } \\
\text { other }\end{array}$ & $\begin{array}{l}17(94.4 .0 \%) \text { white, } 1 \text { (5.6\%) } \\
\text { other }\end{array}$ & $\begin{array}{l}68(89.5 \%) \text { white, } 8 \text { (10.5\%) } \\
\text { other }\end{array}$ & $\begin{array}{l}27(93.1 \%) \text { white, } 2 \text { (6.8\%) } \\
\text { other }\end{array}$ \\
\hline Predementia ID level & $\begin{array}{l}37(39.4 \%) \text { mild, } 47(50 \%) \\
\text { moderate, } 9(9.6 \%) \text { severe, } \\
1(1.2 \%) \text { unknown }\end{array}$ & $\begin{array}{l}6(33.3 \%) \text { mild, } 9(50.0 \%) \\
\text { moderate, } 3(16.7 \%) \text { severe }\end{array}$ & $\begin{array}{l}31(40.8 \%) \text { mild, } 38(50 \%) \\
\text { moderate, } 6(7.9 \%) \text { severe, } 1 \\
(1.3 \%) \text { unknown }\end{array}$ & $\begin{array}{l}13(44.8 \%) \text { mild, } 12(41.4 \%) \\
\text { moderate, } 3(10.3 \%) \text { severe, } \\
1(3.4 \%) \text { unknown }\end{array}$ \\
\hline APOE status & $\begin{array}{l}68(72.3 \%) \text { non-APOE4 carrier, } \\
23(24.5 \%) \text { APOE4 carrier, } 3 \\
(3.2 \%) \text { unknown }\end{array}$ & $\begin{array}{l}12(66.7 \%) \text { non-APOE4 carrier, } \\
5(27.8 \%) \text { APOE4 carrier, } 1 \\
(5.5 \%) \text { unknown }\end{array}$ & $\begin{array}{l}56(73.7 \%) \text { non-APOE4 carrier, } \\
18(23.7 \%) \text { APOE4 carrier, } 2 \\
(2.6 \%) \text { unknown }\end{array}$ & $\begin{array}{l}22(75.9 \%) \text { non-APOE4 } \\
\text { carriers, } 6(20.7 \%) \text { APOE4 } \\
\text { carrier, } 1 \text { (3.4\%) unknown }\end{array}$ \\
\hline $\begin{array}{l}\text { NF-L level, ng/L, } \\
\text { median (range) }\end{array}$ & $22.74(6.11-136.91)$ & $63.76(15.21-136.91)$ & $19.96(6.11-116.84)$ & $32.67(12.23-481.97)$ \\
\hline
\end{tabular}


experienced an occlusive cerebrovascular event 4-6 months prior to donating the blood sample and converted to dementia status at follow-up but was an outlier with an NF-L level of $481.97 \mathrm{ng} / \mathrm{L}$; thus, this individual was excluded from cross-sectional analysis. Among the remaining 94 individuals, NF-L concentration had a median value of $22.74 \mathrm{ng} / \mathrm{L}$ (range $6.11-136.91 \mathrm{ng} / \mathrm{L}$ ). At baseline, 18 of 94 participants had a clinical diagnosis of dementia (Table 1).

\section{NF-L levels and relationship with dementia and other clinical variables}

NF-L levels did not differ by premorbid ID level (KruskalWallis test, $p=0.195$ ), sex (Mann-Whitney $U$ test, $p=0$. 837) or long-standing epilepsy (Mann-Whitney $U$ test, $p=$ 0.858). NF-L level and age of participants were significantly correlated (Spearman's rho $=0.789, p<0.001$ ) (Fig. 1), such that those aged 35 and older had significantly higher levels of NF-L than younger individuals (median $11.52 \mathrm{ng} / \mathrm{L}$ vs. $32.42 \mathrm{ng} / \mathrm{L}$, Mann-Whitney $U$ test $p<0.001$ ).

Those with dementia had significantly higher levels of NF-L (median $63.76 \mathrm{ng} / \mathrm{L}$ vs. $19.96 \mathrm{ng} / \mathrm{L}$; Mann-Whitney $U$ test $p<0.001$ ), and a logistic regression model adjusting for age, sex, and APOE4 status revealed that NF-L levels remained predictive of dementia status $(p=0.022)$.

\section{NF-L and predictive validity for dementia}

Seven (24.1\%) of 29 individuals with follow-up cognitive data had a clinical diagnosis of dementia at baseline, all with the dementia diagnosis retained at follow-up, with a further $2(6.9 \%)$ individuals converting to dementia status by follow-up, whereas $3(10.3 \%)$ participants were under investigation for dementia at follow-up. Predictive validity of NF-L levels at baseline was explored by combining individuals with confirmed or suspected dementia at follow-up ( $n=12$, median NF-L $77.38 \mathrm{ng} / \mathrm{L})$ and comparing them with those who remained dementia-free (median NF-L $19.94 \mathrm{ng} / \mathrm{L}$ ). Higher levels of NF-L at baseline predicted the likelihood of dementia at followup, even when adjusted for age and sex $(p=0.036)$.

\section{Discussion}

We have demonstrated that NF-L measured in blood using an ultrasensitive assay is strongly associated with age and dementia status in individuals with DS, and baseline levels were predictive of dementia diagnosis over time. Furthermore, NF-L levels did not differ according to severity of premorbid ID or by long-standing epilepsy diagnosis (a common neurological comorbidity in DS), suggesting that it is a stable and feasible biomarker that can be used in clinical populations.

Our results indicate that this marker could pinpoint the onset of neurodegeneration in DS. NF-L showed an age relationship in keeping with postmortem data and amyloid positron emission tomography studies of $\mathrm{AD}$ pathology in adults with DS $[1,14,15]$. In familial AD, serum NF-L concentration is increased prior to symptom onset and correlates with measures of disease stage and severity [10]. In sporadic AD, plasma NF-L concentration is increased already in the mild cognitive impairment stage of the

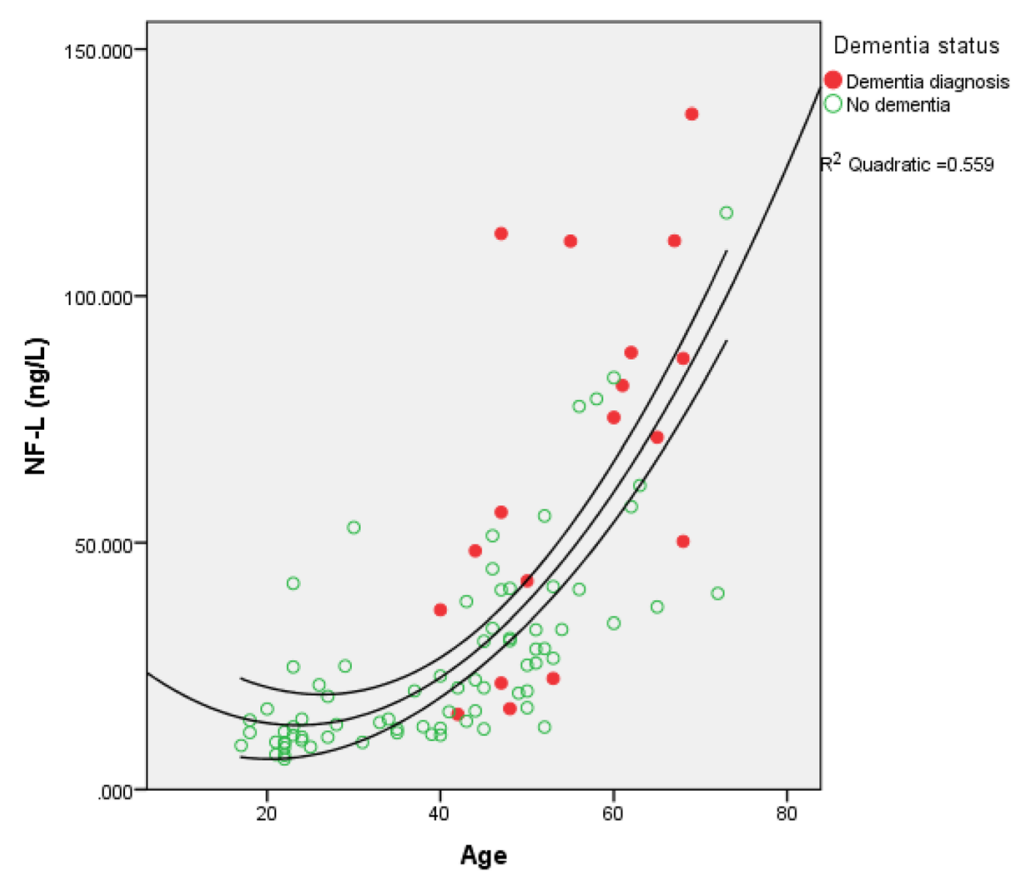

Fig. 1 Neurofilament light (NF-L) concentration by age and dementia status of individuals with Down syndrome 
disease and correlates with cognitive hallmarks of the disease [9]. Our results show that NF-L has similar relationships with clinical dementia diagnoses in DS. Substantial neurodegeneration associated with the buildup of amyloid likely occurs before a threshold for dementia diagnosis is reached, and like the presence of amyloid plaques preceding the clinical signs by several decades, NF-L levels may be increased some time before onset of significant symptoms, in keeping with some degree of neurodegeneration prior to clinical impact. Further longitudinal studies may elucidate the relationship between NF-L levels and development of symptoms in genetically predisposed populations such as DS.

Although NF-L is a marker of axonal damage, and thus not specific to AD [7-9, 16], in a population such as $\mathrm{DS}$ where $\mathrm{AD}$ is almost always the cause of dementia, the lack of specificity is arguably less of an issue, and NF-L could potentially be used as a biomarker in treatment trials. Recent studies in rodent models of neurodegenerative diseases showed that NF-L levels in CSF and plasma responded to experimental manipulation and targeted therapy [17], and normalization of serum/plasma NF-L in response to treatment has already been demonstrated in patients with multiple sclerosis $[8,18]$. These findings suggest that NF-L may be a useful biomarker of response to treatment.

The relationship between serum NF-L levels and genetic markers and other AD biomarkers, including markers of inflammation and oxidative stress (both of which are increased in DS and associated with cognitive decline [19-22]), could also be explored in future research to refine prediction of cognitive decline and to stratify patients with Down syndrome according to dementia risk for clinical trials of potential treatments.

\section{Conclusions}

Although further work is required to establish long-term predictive and concurrent validity of NF-L, our data suggest that this biomarker could be instrumental in allowing an experimental medicine approach in individuals with DS and other high-risk populations to test treatments that might prevent or delay dementia onset.

\footnotetext{
Acknowledgements

The authors thank all the participants in this study for their time. $\mathrm{HZ}$ is a Wallenberg Academy Fellow and receives support from the European Research Council, the Swedish Research Council, and Frimurarestiftelsen. $\mathrm{HZ}$ is further supported by the Wolfson Foundation, the Wellcome Trust, and the UK Dementia Research Institute. DN is also supported by the National Medical Research Council Singapore (NMRC/CIRG/1438/2015) and the Singapore Ministry of Education AcRF-Tier2 (2015-T2-1-023). This research was further supported by the National Institute for Health Research networks (mental health, dementias, and neurology) and participating NHS trusts. We thank our NHS network of sites that helped to identify participants.

LonDownS Consortium principal investigators: Andre Strydom (chief investigator), Department of Forensic and Neurodevelopmental Sciences, Institute of Psychiatry, Psychology and Neuroscience, King's College London, London, UK, and Division of Psychiatry, University College London, London,
}

UK; Elizabeth Fisher, Department of Neurodegenerative Disease, UCL Institute of Neurology, London, UK; Dean Nizetic, Blizard Institute, Barts and the London School of Medicine, Queen Mary University of London, London, UK, and Lee Kong Chian School of Medicine, Nanyang Technological University, Singapore; John Hardy, Reta Lila Weston Institute, Institute of Neurology, University College London, London, UK, and UK Dementia Research Institute at UCL, London, UK; Victor Tybulewicz, Francis Crick Institute, London, UK, and Department of Medicine, Imperial College London, London, UK; and Annette Karmiloff-Smith (Birkbeck University of London, London, UK, deceased). Other members of The LonDownS Consortium who contributed to data collection are Sarah Hamburg and Rosalyn Hithersay (both Department of Forensic and Neurodevelopmental Sciences, Institute of Psychiatry, Psychology and Neuroscience, King's College London, London, UK, and Division of Psychiatry, University College London, London, UK).

\section{Funding}

This work was funded by a Wellcome Trust Strategic Award (grant number 098330/Z/12/Z) conferred upon The London Down Syndrome (LonDownS) Consortium (Chief Investigator, Andre Strydom). The funder had no role in study design; the collection, analysis, or interpretation of the data; the writing of the report; or the decision to submit the report for publication.

\section{Availability of data and materials}

The datasets used and/or analyzed during the present study are available from the corresponding author on reasonable request.

\section{Authors' contributions}

AS and $\mathrm{HZ}$ conceived of and designed the study. $\mathrm{AH}, \mathrm{KYM}, \mathrm{JH}, \mathrm{JG}, \mathrm{DN}$, and $\mathrm{HZ}$ acquired data. AS and CMS analyzed data. AS and CMS wrote the manuscript. All coauthors revised the manuscript for important intellectual content. All authors read and approved the final manuscript.

\section{Ethics approval and consent to participate}

The North West Wales National Health Service (NHS) Research Ethics Committee provided ethical approval for a longitudinal study of cognitive ability and dementia in DS (13/WA/0194). For those with decision-making capacity, written consent was obtained, whereas for those who did not have decision-making capacity, a consultee indicated their agreement to participation.

\section{Competing interests}

AS has consulted for Ono Pharmaceutical Co., is an adviser to the UK Down Syndrome Association, and is an advisory board member of the LuMind Research Down Syndrome Foundation (USA). HZ has served on advisory boards of Roche Diagnostics, Eli Lilly, and Teva and is a cofounder of Brain Biomarker Solutions with Gothenburg AB, a GU Ventures-based platform company at the University of Gothenburg. The other authors declare that they have no competing interests.

\section{Publisher's Note}

Springer Nature remains neutral with regard to jurisdictional claims in published maps and institutional affiliations.

\section{Author details}

${ }^{1}$ Department of Forensic and Neurodevelopmental Sciences, Institute of Psychiatry, Psychology and Neuroscience, King's College London, 16 De Crespigny Park, London SE5 8AF, UK. Division of Psychiatry, University College London, London, UK. ${ }^{3}$ The LonDownS Consortium, Department of Forensic and Neurodevelopmental Sciences, Institute of Psychiatry, Psychology and Neuroscience, King's College London, Denmark Hill, London SE5 8AF, UK. ${ }^{4}$ Department of Molecular Neuroscience, Institute of Neurology, University College London, London, UK. ${ }^{5}$ Division of Life Science, Hong Kong University of Science and Technology, Hong Kong, Hong Kong, Special Administrative Region of China. ${ }^{6}$ Reta Lila Weston Institute, Institute of Neurology, University College London, London, UK. ${ }^{7}$ Blizard Institute, Barts and the London School of Medicine, Queen Mary University of London, London, UK. ${ }^{8}$ Lee Kong Chian School of Medicine, Nanyang Technological University, Singapore, Singapore. ${ }^{9}$ Department of Psychiatry and Neurochemistry, Institute of Neuroscience and Physiology, The Sahlgrenska Academy at the University of Gothenburg, Gothenburg, Sweden. ${ }^{10}$ UK Dementia Research Institute at UCL, London, UK. 
Received: 16 February 2018 Accepted: 14 March 2018 Published online: 10 April 2018

\section{References}

1. Wiseman FK, Al-Janabi T, Hardy J, Karmiloff-Smith A, Nizetic D, Tybulewicz VL, Fisher EM, Strydom A. A genetic cause of Alzheimer disease: mechanistic insights from Down syndrome. Nat Rev Neurosci. 2015;16(9):564-74.

2. McCarron M, McCallion P, Reilly E, Dunne P, Carroll R, Mulryan N. A prospective 20-year longitudinal follow-up of dementia in persons with Down syndrome. J Intellect Disabil Res. 2017;61(9):843-52.

3. Sinai A, Mokrysz C, Bernal J, Bohnen I, Bonell S, Courtenay K, Dodd K, Gazizova D, Hassiotis A, Hillier R, et al. Predictors of age of diagnosis and survival of Alzheimer's disease in down syndrome. J Alzheimers Dis. 2018; 61(2):717-28.

4. Zis P, Strydom A. Clinical aspects and biomarkers of Alzheimer's disease in Down syndrome. Free Radic Biol Med. 2018;114:3-9.

5. Zetterberg H. Neurofilament light: a dynamic cross-disease fluid biomarker for neurodegeneration. Neuron. 2016;91(1):1-3.

6. Gisslen M, Price RW, Andreasson U, Norgren N, Nilsson S, Hagberg L, Fuchs D, Spudich S, Blennow K, Zetterberg H. Plasma concentration of the neurofilament light protein (NFL) is a biomarker of CNS injury in HIV infection: a cross-sectional study. Ebiomedicine. 2016;3:135-40.

7. Rohrer JD, Woollacott IO, Dick KM, Brotherhood E, Gordon E, Fellows A, Toombs J, Druyeh R, Cardoso MJ, Ourselin S, et al. Serum neurofilament light chain protein is a measure of disease intensity in frontotemporal dementia. Neurology. 2016;87(13):1329-36.

8. Disanto G, Barro C, Benkert P, Naegelin Y, Schadelin S, Giardiello A, Zecca C, Blennow K, Zetterberg H, Leppert D, et al. Serum neurofilament light: a biomarker of neuronal damage in multiple sclerosis. Ann Neurol. 2017;81(6): 857-70.

9. Mattsson N, Andreasson U, Zetterberg H. Blennow K; Alzheimer's Disease Neuroimaging Initiative. Association of plasma neurofilament light with neurodegeneration in patients with Alzheimer disease. JAMA Neurol. 2017; 74(5):557-66.

10. Weston PSJ, Poole T, Ryan NS, Nair A, Liang Y, Macpherson K, Druyeh R,

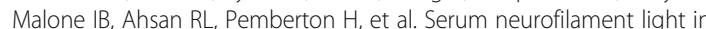
familial Alzheimer disease: a marker of early neurodegeneration. Neurology. 2017:89(21):2167-75

11. Startin CM, Hamburg S, Hithersay R, Davies A, Rodger E, Aggarwal N, AlJanabi T, Strydom A. The LonDownS adult cognitive assessment to study cognitive abilities and decline in Down syndrome. Wellcome Open Res. 2016;1:11

12. Ball SL, Holland AJ, Huppert FA, Treppner P, Watson P, Hon J. The modified CAMDEX informant interview is a valid and reliable tool for use in the diagnosis of dementia in adults with Down's syndrome. J Intellect Disabil Res. 2004;48(Pt 6):611-20.

13. Strydom A, Hassiotis A, King M, Livingston G. The relationship of dementia prevalence in older adults with intellectual disability (ID) to age and severity of ID. Psychol Med. 2009;39(1):13-21.

14. Annus T, Wilson LR, Hong YT, Acosta-Cabronero J, Fryer TD, CardenasBlanco A, Smith R, Boros I, Coles JP, Aigbirhio Fl, et al. The pattern of amyloid accumulation in the brains of adults with Down syndrome. Alzheimers Dement. 2016;12(5):538-45.

15. Hartley SL, Handen BL, Devenny DA, Hardison R, Mihaila I, Price JC, Cohen AD, Klunk WE, Mailick MR, Johnson SC, et al. Cognitive functioning in relation to brain amyloid- $\beta$ in healthy adults with Down syndrome. Brain. 2014;137(Pt 9):2556-63.

16. Lu CH, Macdonald-Wallis C, Gray E, Pearce N, Petzold A, Norgren N, Giovannoni G, Fratta P, Sidle K, Fish M, et al. Neurofilament light chain: a prognostic biomarker in amyotrophic lateral sclerosis. Neurology. 2015; 84(22):2247-57.

17. Bacioglu M, Maia LF, Preische O, Schelle J, Apel A, Kaeser SA, Schweighause M, Eninger T, Lambert M, Pilotto A, et al. Neurofilament light chain in blood and CSF as marker of disease progression in mouse models and in neurodegenerative diseases. Neuron. 2016;91(1):56-66.

18. Piehl F, Kockum I, Khademi M, Blennow K, Lycke J, Zetterberg H, Olsson T. Plasma neurofilament light chain levels in patients with MS switching from injectable therapies to fingolimod. Mult Scler. 2017. https://doi.org/10.1177/ 1352458517715132
19. Zis P, Dickinson M, Shende S, Walker Z, Strydom A. Oxidative stress and memory decline in adults with Down syndrome: longitudinal study. J Alzheimers Dis. 2012;31(2):277-83.

20. Zis P, McHugh P, McQuillin A, Pratico D, Dickinson M, Shende S, Walker Z, Strydom A. Memory decline in Down syndrome and its relationship to iPF2a, a urinary marker of oxidative stress. PLoS One. 2014;9(6):e97709.

21. Zis P, Strydom A, Buckley D, Adekitan D, McHugh PC. Cognitive ability in Down syndrome and its relationship to urinary neopterin, a marker of activated cellular immunity. Neurosci Lett. 2017;636:254-7.

22. Iulita MF, Ower A, Barone C, Pentz R, Gubert P, Romano C, Cantarella RA, Elia F, Buono S, Recupero M, et al. An inflammatory and trophic disconnect biomarker profile revealed in Down syndrome plasma: relation to cognitive decline and longitudinal evaluation. Alzheimers Dement. 2016;12(11):1132-48.

\section{Submit your next manuscript to BioMed Central and we will help you at every step:}

- We accept pre-submission inquiries

- Our selector tool helps you to find the most relevant journal

- We provide round the clock customer support

- Convenient online submission

- Thorough peer review

- Inclusion in PubMed and all major indexing services

- Maximum visibility for your research

Submit your manuscript at www.biomedcentral.com/submit 